Technological University Dublin ARROW@TU Dublin

\title{
A Comparison of Raman, FTIR and ATR-FTIR Micro Spectroscopy for Imaging Human Skin Tissue Sections.
}

\author{
Syed Mehmood Ali \\ Technological University Dublin \\ Franck Bonnier \\ Technological University Dublin, Franck.Bonnier@tudublin.ie \\ Helen Lambkin \\ Technological University Dublin
}

See next page for additional authors

Follow this and additional works at: https://arrow.tudublin.ie/biophonart

Part of the Analytical, Diagnostic and Therapeutic Techniques and Equipment Commons, and the Physics Commons

\section{Recommended Citation}

Ali, S.M. et al (2013) A comparison of Raman, FTIR and ATR-FTIR micro spectroscopy for imaging human skin tissue sections. Analytical Methods, 5, pp.2281-2291.

This Article is brought to you for free and open access by the DIT Biophotonics and Imaging at ARROW@TU Dublin. It has been accepted for inclusion in Articles by an authorized administrator of ARROW@TU Dublin. For more information, please contact arrow.admin@tudublin.ie, aisling.coyne@tudublin.ie,gerard.connolly@tudublin.ie. Funder: PRTLI Cycle 4 NBIPI

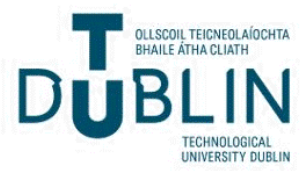




\section{Authors}

Syed Mehmood Ali, Franck Bonnier, Helen Lambkin, Kathleen Flynn, V McDonagh, Claragh Healy, T C. Lee, Fiona Lyng, and Hugh Byrne 


\section{A comparison of Raman, FTIR and ATR-FTIR micro spectroscopy for imaging human skin tissue sections}

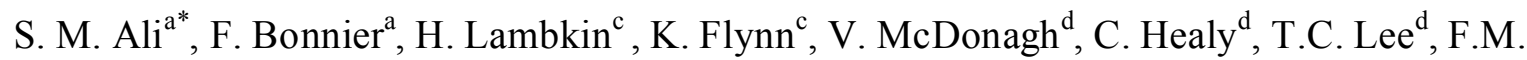

Lyng $^{\mathrm{a}}$, H.J. Byrne

${ }^{a}$ Radiation and Environmental Science Centre, Focas Research Institute, Dublin Institute of Technology, Kevin Street, Dublin 8, Ireland.

${ }^{\mathrm{b}}$ School of Biological Sciences, Dublin Institute of Technology, Kevin Street, Dublin 8, Ireland.

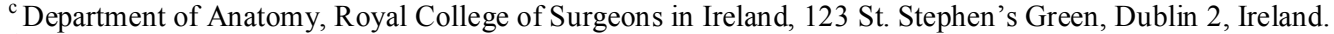

${ }^{\mathrm{d}}$ Focas Research Institute, Dublin Institute of Technology, Kevin Street, Dublin 8, Ireland.

*Corresponding Author: Syed Mehmood Ali,

Radiation and Environmental Science Centre

Focas Research Institute, Dublin Institute of Technology,

Kevin Street, Dublin 8, Ireland.

E mail address: Mehmood.ali@dit.ie

Ph: +35314027966 


\begin{abstract}
Raman and Infrared absorption spectroscopies are compared for the analysis of human hand skin tissue sections. The tissue sections have been formalin fixed and paraffin processed, and subsequently dewaxed. Fourier Transform infrared (FTIR) spectra are preprocessed using the resonant Mie - extended multiplicative scattering algorithm to remove spectral artefacts. FTIR images of resolution $4 \mathrm{~cm}^{-1}$, analysed using K-means cluster analysis, reveal the double layer structure of the dermis and epidermis, but no further layer differentiation is achieved using the higher spatial resolution of the Attenuated Total Reflection imaging or improved spectral resolution of $2 \mathrm{~cm}^{-1}$. At comparable spectral and spatial resolutions and measurement on the same samples, Raman scattering produces spectra of significantly higher spectral detail and can differentiate the stratum corneum from the underlying epithelial layer, and, in the absence of melanin in an artificial skin model, can further differentiate the basal layer from the overlying epithelium. The differences in the performance of the techniques are therefore not instrumentational and are discussed in terms of the technological and fundamental differences between the two complementary techniques.
\end{abstract}

\title{
Keywords
}

Fourier Transform Infrared Spectroscopy, Attenuated Total Reflection Microscopy, Raman Spectroscopy, K-Means Cluster Analysis, Skin Tissue, Artificial Skin model, Diagnostics 


\section{Introduction}

Vibrational spectroscopic techniques, both Raman and Infrared absorption, have significant potential in the field of biomedical analysis, as they can give spatially resolved biochemical information without the use of extrinsic labels and without being invasive or destructive to the system studied. Both techniques are truly label-free, since the inherent vibrational signature of the biochemistry of the cell or tissue is being observed ${ }^{1}$. In the past, many studies have been carried out using either Raman or Fourier Transform Infrared absorption (FTIR) spectroscopy to classify tissue with a view to disease diagnosis. Some of the tissue types examined by various groups include cervical ${ }^{2,3}$ skin ${ }^{4-7}$, lung ${ }^{8,9}$, brain ${ }^{10}$, oesophagus ${ }^{11,12}$, colon ${ }^{13}$, prostate ${ }^{14}$, nasopharynx ${ }^{15}$, larynx ${ }^{16}$ oral $^{17}$, breast ${ }^{18,19}$, liver ${ }^{20}$. However, although often referred to as complementary techniques, Raman and FTIR spectroscopy are based on very different fundamental physical phenomena, and thus present different relative advantages and disadvantages, and indeed technical challenges to their clinical implementation.

Raman spectroscopy, a scattering technique, measures the shift of frequency as a result of the exchange of energy between the incident photon and the material vibrations (or rotations). As such, it can occur nonresonantly and can be measured across the spectral range, although it is most commonly measured in the UV, visible and NIR regions. IR spectroscopy relies on the direct absorption of light as a result of transitions between vibrational (or rotational) states and thus must be carried out in the (usually mid) IR region of the spectrum. Whereas the IR electric dipole transitions rely on the change in the average dipole moment of the vibration as a result of the transition, Raman scattering relies on a nonzero change in the average polarisability. IR cross sections therefore tend to be strongest in asymmetric polar moieties, whereas Raman cross sections tend to be strong for symmetric, electron rich moieties. Water is therefore a very strong 
IR absorber but is relatively a weak Raman scatterer, which suggests Raman as the technique of choice for in vivo applications. The fundamental differences also lead to distinct technological considerations in the application of the two techniques. Raman is an inherently weak technique, and profiling large areas by point to point mapping of signals acquired over (often) tens of seconds is time consuming. Recently developed multidetector arrays for FTIR microscopy allow true imaging of large surface areas in minutes. However, as it operates in the UV, visible and NIR regions, Raman intrinsically offers sub micrometer spatial resolution in air, whereas the diffraction limit for FTIR is wavelength limited to $5-10 \mu \mathrm{m}$. Raman spectroscopy is therefore the technique of preference for subcellular profiling ${ }^{21-24}$, whereas FTIR is more suitable for large area profiling ${ }^{25,26}$. More recent advances in Attenuated Total Reflection (ATR) microscopic imaging offer the potential of FTIR spatial resolution comparable to that of optical wavelengths, however ${ }^{27,28}$. In each case, the spectral resolution is determined by the instrument used. Common benchtop FTIR spectrometers have a variable resolution, of $0.5-32 \mathrm{~cm}^{-1}$, determined by the path length difference of the interfering beams, in turn determined by the distance travelled by the moving mirror. For gas phase work, off the shelf high resolution instruments can routinely go to $0.0009 \mathrm{~cm}^{-1}$. Dispersive Raman spectrometers have spectral dispersion, determined by the diffraction grating, spectrometer length and the pixel size of the detector array, of $\sim 1-20 \mathrm{~cm}^{-1}$ per pixel, but can be as low as $0.25 \mathrm{~cm}^{-1}$ in some commercial instruments.

Independent of the technologies, both Raman and FTIR spectroscopic analyses of physically and chemically inhomogeneous bio-samples suffer from significant effects which can obscure or distort the spectral data. As Raman scattering is a weak effect, and commonly measured on the Stokes (longer wavelength) side of the excitation wavelength, any fluorescence from the sample can completely swamp the Raman spectrum. Even in nonabsorbing (and therefore 
nonfluorescent) samples, a significant background can arise due to stray light from noncollimated elastic scattering of the source laser or the Raman bands themselves ${ }^{29}$. Operating at the longer wavelengths of the NIR can alleviate both effects, and, in immersion, the fluid acts as an optical clearing fluid reducing the scattering background to almost zero ${ }^{23}$. For live cell analysis, it has been shown that measurement in immersion of cells grown in a collagen matrix effectively reduces the background and substrate contributions to that of water, greatly simplifying preprocessing routines and increasing confidence in the data to be analysed. FTIR spectroscopy of cells and tissues has been plagued by the so-called "dispersion artefact" for a number of years ${ }^{30-33}$. More recently it has been demonstrated that the effects, which can give rise to a broad undulating background and a distortion of the absorption bands, most characteristically the amide I band, have origin in the resonant reflection ${ }^{34}$ and scattering ${ }^{35}$ contributions to the measured absorption, in either transmission or transflection mode. In order to correct for the dispersion artefacts, the extended multiplicative scattering correction (EMSC) has been further evolved ${ }^{36}$ such that routine measurements can be pre-processed to data which truly reflect the biochemical variations of the sample.

Technological and data processing advances for both Raman and FTIR spectroscopy in recent years have therefore enhanced the suitability for each in biomedical screening applications as well as the confidence in the data produced. Many groups have explored the applications of the two techniques in parallel, but there are few studies which directly compare the performance of the two techniques on the same samples. In this study, both techniques will be employed to explore the biochemical profiling of the same human skin tissue sections. The results are compared and contrasted in terms of the spatial and spectral information obtained. Generally, it is well accepted that Raman provides better spatial and spectral resolution than IR, in common 
commercially available systems. However, few studies have been performed on the same samples to directly compare the performance. Although the differences in the results are often ascribed to instrumental or technical limitations, this study demonstrates that, at comparable spatial and spectral resolution, Raman provides significantly richer spectral information and better spatial differentiation of the tissue structure, indicating the importance of the differences in the fundamental physical principles of the techniques.

\section{Materials and Methods}

\section{Tissue samples}

Human skin tissue was provided through the Anatomical Gift Programme of the Royal College of Surgeons of Ireland (RCSI). For this study, dorsal skin sections from human hand were employed.

MatTek's patented EpiDerm ${ }^{\mathrm{TM}}$ artificial skin model consists of normal, human-derived epidermal keratinocytes (NHEK). The EpiDerm cultures should be treated similarly to normal in vitro cell cultures. On delivery, the samples are transferred into the medium supplied and are incubated for 24 hours in $\mathrm{CO}_{2}$ at $37^{\circ} \mathrm{C}$.

For sectioning, both human cadaver and EpiDerm tissue sections were formalin fixed paraffin processed (FFPP), and subsequently dewaxed using xylene. The skin and EpiDerm tissues were automatically processed (Leica Histokinette 2000) to wax blocks using four principle steps, as follows: 
(i) Vacuum fixation in $10 \%$ buffered formal saline histograde ph 6.8-7.2 (J.T Baker, Deventer, the Netherlands) and heating to $30^{\circ} \mathrm{C}$.

(ii) Vacuum dehydration in industrial methylated spirit IMS T100 (Lennox, Dublin Ireland)

(iii) Vacuum clearing in xylene (Serosep, Limerick, Ireland) and heating to $35^{\circ} \mathrm{C}$.

(iv) Vacuum impregnation with tissue 111 Embedding Wax with polymer added (Sakura, Zoeterwoude, The Netherlands) and heating to $59^{\circ} \mathrm{C}$.

After wax impregnation, tissue was embedded and sliced into $20 \mu \mathrm{m}$ sections using a microtome, mounted on a $\mathrm{CaF}_{2}$ substrate and dried. To remove the wax, the samples were immersed in a series of baths consisting of two baths of xylene (BDH, Dorest, UK) for five minutes and four minutes respectively, two baths of Ethanol Absolute (Merck, Dorest, UK) for three minutes and two minutes and a final bath of Industrial Methylated spirits 95\% (Lennox Dublin, Ireland) for one minute. Cross sections of $20 \mu \mathrm{m}$ thickness were cut with a microtome (Leica CM $1850 \mathrm{UV}$ ).

\section{FTIR Instrumentation}

IR absorption measurements in this work were carried out using a Perkin Elmer Spotlight 400N FTIR imaging system. The system is also equipped with an AutoImage microscope system operating with a x 40 Cassegrain objective, and can operate in transmission or reflection mode. FTIR images were acquired in transmission and ATR imaging modes. The data were collected over the nominal free-scanning spectral range with an interferometer speed of $1.0 \mathrm{~cm} / \mathrm{s}$ and collected using a liquid nitrogen cooled mercury cadmium telluride (MCT-A) line detector. For transmission mode, spectral measurements were acquired with a pixel size of $6.25 \mu \mathrm{m}$ x $6.25 \mu \mathrm{m}$ 
at a spectral resolution of $4 \mathrm{~cm}^{-1}$. Background measurements were acquired on a region with no tissue with 120 scans per pixel whereas 32 scans per pixel were recorded from the sample.

ATR images were recorded with the Perkin Elmer Spotlight 400N ATR imaging adapter using a germanium crystal objective of dimensions $\sim 600 \mu \mathrm{m} \times 600 \mu \mathrm{m}$ placed in direct contact with the sample. In ATR mode, spectral images were acquired with a pixel size of $1.56 \mu \mathrm{m} \times 1.56 \mu \mathrm{m}$, with 32 scans per pixel at a spectral resolution of $8 \mathrm{~cm}^{-1}$ or $2 \mathrm{~cm}^{-1}$. ATR images were acquired across a $400 \mu \mathrm{m} \times 100 \mu \mathrm{m}$ region of the ATR crystal. The ATR crystal was gently placed in contact with the tissue using minimal pressure to ensure good contact and minimize tissue damage. No obvious damage to the tissue was observable using this approach.

\section{Raman Instrumentation}

A Horiba Jobin-Yvon LabRAM HR800 spectrometer with an external $300 \mathrm{~mW}$ diode laser operating at $785 \mathrm{~nm}$ as source was used throughout this work. For the measurements, a x100 immersion objective (LUMPlanF1, Olympus) was employed, providing a spot size of $\sim 1 \mu \mathrm{m}$ at the sample. The confocal hole was set at $100 \mu \mathrm{m}$ for all measurements, the specified setting for confocal operation. The system was spectrally calibrated to the $520.7 \mathrm{~cm}^{-1}$ spectral line of silicon. The LabRAM system is a confocal spectrometer that contains two interchangeable gratings (300 and 900 lines/mm respectively). In the following experiments, the 300 lines $/ \mathrm{mm}$ grating was used, giving a spectral dispersion of $\sim 1.5 \mathrm{~cm}^{-1}$ per pixel. The detector used was a 16bit dynamic range Peltier cooled CCD detector. A step size of $2 \mu \mathrm{m}$ was employed for tissue mapping. 
Tissue samples were measured under water immersion to minimise the spectral background ${ }^{23}$, and spectra were recorded using the immersion objective. Once all spectra were acquired, a background of substrate measured under identical conditions was subtracted. Minimal baseline correction, smoothing and normalization were also performed in order to improve the quality of the acquired spectra.

\section{Data analysis}

The different data analysis steps were performed using Matlab (Mathworks, USA). The resonant Mie scattering correction algorithm (RMieS-EMSC) was employed to remove scattering effects $34,35,37,38$, as has been successfully demonstrated in a number of studies ${ }^{39,40}$. The algorithm utilises a scatter-free reference spectrum $\left(Z_{\text {Ref }}\right)$, to correct the raw tissue spectrum $\left(Z_{\text {Raw }}\right)$. In the current work, a Matrigel (a commercial artificial extra cellular matrix that contains all the key components expected in tissue) spectrum was employed as reference. Other variable parameters for the algorithm include the number of iterations: 20, number of principle components: 7, Mie theory option: Resonant Mie (RMieS) correction, lower range of the scattering particle radius: 2 $\mu \mathrm{m}$, Upper range of the scattering particle radius: $8 \mu \mathrm{m}$, lower range of scattering particle average refractive index: 1.1 , and upper range of scattering particle average refractive index: 1.5 .

After correction, K-means cluster analysis (KMCA) was employed to analyse the spectral variations in the tissue. It is one of the simplest unsupervised learning algorithms that solves the well known clustering problem and is often used for spectral image analysis ${ }^{41}$, In general, clustering is the partitioning of a data set into subsets (clusters) so that the differences between the data within each cluster are minimized and the differences between clusters are maximized according to some defined distance measure. Using KMCA, the large amount of data in a 
spectral map can be reduced to mean spectra and the spatial distribution can easily be visualised. The spectral data were used as inputs to KMCA. The clustering analysis algorithm was used to find groups of spectra with similar spectral characteristics (clusters), each one representing regions of the image with similar biochemical profiles.

KMCA can be used for the identification of different structures and classification of tumoral regions in tissue sections by Raman and FTIR spectroscopy ${ }^{42,43}$. However, for the analysis of the spectral response of the tissue sections, the spectra recorded on the edge of the tissue exhibit a high degree of variability due to the transition from the outer layer of tissue to the substrate. After the cluster analysis, the variability between these spectra can interfere with the analysis, resulting in the creation of new clusters. Thus, in order to the best visualize the different structures existing within the tissue; the number of clusters was increased to 5 to yield the best reproducibility. In this way, the variability in the spectra obtained at the edge of the tissue is contained in different clusters and does not interfere with the identification of different structures present in the tissue. After analysis, the spectra were assigned to the 5 different groups according to their similarities and a colour attributed to each cluster. False colour maps were constructed representing the partition of the different clusters in the tissue.

\section{Results}

\section{Human skin tissue sections}

Figure 1A shows an optical microscopic image of a dewaxed human hand skin section of $20 \mu \mathrm{m}$ thickness. Within the region isolated within the overlaid square, the optical image appears to have a layered structure, with a more complex morphology in the lower left quadrant. Figure 1B shows a map of the mean FTIR spectral absorbance, mapped in the same region using the 
transmission microscopic mode of the Spotlight 400N. In both $\mathrm{X}$ and $\mathrm{Y}$ directions, the pixel size is $6.25 \mu \mathrm{m}$, and thus the image size is $250 \mu \mathrm{m} \times 181 \mu \mathrm{m}$. Total acquisition time was $\sim 5 \mathrm{hrs}$. Figure $1 \mathrm{C}$ shows the corresponding KMCA map of the spectral data and the mean spectra of clusters 5 , 3 and 1 are shown in figure 2A. Cluster 2 and 4 represent the image background and cluster 1 and 3 are identical in spectral profile and similar in spatial distribution. Notably, the cluster mean spectra are characteristically distorted ${ }^{35}$, showing a sloping baseline between 2000 and $4000 \mathrm{~cm}^{-}$ ${ }^{1}$ and a more pronounced decrease in intensity on the higher wavenumber side of the amide I band at $\sim 1645 \mathrm{~cm}^{-1}$. The broad variation of the baseline has been associated with classic (nonresonant) Mie scattering, while the pronounced dip in absorbance at $\sim 1724 \mathrm{~cm}^{-1}$ is predominantly due to resonant scattering and/or reflection ${ }^{34,} 35$. The spectral data set were therefore preprocessed using the RMieS-EMSC correction algorithm, using Matrigel as reference, and 20 iterations, a process which took $\sim 9 \mathrm{hrs}$. The KMCA map of the preprocessed data is shown in Figure 1D, while the mean cluster spectra are shown in Figure 2B (fingerprint region only in Figure 2C). In addition to recovering an almost flat baseline, the RMieS-EMSC correction has removed the distortion of the amide I band. In all following sections, all FTIR spectral data sets were subject to correction using the RMie - EMSC scattering correction using 20 iterations.

Having removed the spectral distortions of the so-called "dispersion artefacts", the KMCA map of Figure 1D should represent the biochemical profile of the skin tissue section. Cluster 2 and 5 contain no biological features and are spatially associated with the substrate and are therefore not plotted. The mean spectrum of Cluster 3, plotted in Figure 2B (i) appears to represent a biological profile, but comparison of Figure 1D with the average absorbance map of Figure 1B shows that cluster 3 maps a region of low absorbance at the edge of the tissue section and thus should be considered as an edge effect rather than a distinct layer of the tissue section. The 
biochemical profile is therefore represented by clusters 1, plotted in Figure 2B (ii) and 4, plotted in Figure 2B (iii) and the distinct layers are associated with the epidermal and dermal layers. Qualitatively, the character of the $\mathrm{CH}_{2}$ and $\mathrm{CH}_{3}$ bands between $2800 \mathrm{~cm}^{-1}$ and $3000 \mathrm{~cm}^{-1}$ in the dermis is different from the epidermis. Both the absolute intensity of the $\mathrm{CH}_{2}$ absorption and the ratio of the $\mathrm{CH}_{2}$ and $\mathrm{CH}_{3}$ intensities are decreased in the dermis. These features are associated with lipids, which, although largely removed by the tissue processing, are present in higher quantities in the skin epidermis than the dermis ${ }^{44}$. A strong shoulder at $1644 \mathrm{~cm}^{-1}$ (collagen) is prominent in the mean spectrum of the dermis. A series of absorption bands attributed to collagen $\left(1336 \mathrm{~cm}^{-1}, 1280 \mathrm{~cm}^{-1}, 1206 \mathrm{~cm}^{-1}, 1082 \mathrm{~cm}^{-1}\right.$ and $\left.1030 \mathrm{~cm}^{-1}\right)$ are also present in the spectrum of the dermis but not that of the epidermis.

FTIR spectroscopic microscopy can therefore successfully differentiate the layer structure of the skin section based on the biochemical signatures. In comparison, however, Raman spectroscopy provides a much clearer demarkation of the layer structure of skin tissue and significantly more spectral information. Figure 3A shows an optical microscopic image of a similar $20 \mu \mathrm{m}$ thick dewaxed human hand tissue section. Again a layered morphology is apparent. Figure 3B shows a 5 cluster KMCA map of the region of tissue indicated by the rectangle of figure $3 \mathrm{~A}$, mapped by Raman spectroscopy. Each pixel corresponds to $2 \mu \mathrm{m}$ in both $\mathrm{X}$ and $\mathrm{Y}$ directions, resulting in an image size of $36 \mu \mathrm{m} \times 200 \mu \mathrm{m}$. The mean spectra corresponding to clusters 3,5 and 4 are shown in figure $3 \mathrm{C}$ (fingerprint region only). Clusters 1 and 2 correspond to the substrate.

A detailed analysis of the Raman maps of unprocessed and processed skin tissue sections has recently been presented ${ }^{44}$. The region represented by cluster 4 is associated with the dermal layer of the skin sample based on the dominant contributions of collagen. The intermediate layer characterised by cluster 5 is dominated by fluorescence from melanin, which has been removed 
by background subtraction in figure 3 , identifying it with the basal and malpighian layers, while cluster 3 is associated with the stratum corneum, based on the strong features of keratinocytes.

\section{Epiderm skin model}

The structure of the EpiDerm skin model is similar to natural skin. Notably, it does not contain a melanin rich basal layer, which presents the advantage that its fluorescence will not interfere with the Raman spectra, and the pure Raman profile of the epidermis can be revealed. Figure 4A shows an optical microscopic image of the dewaxed model skin section of $20 \mu \mathrm{m}$ thickness. As for the human skin section, three different regions are visually apparent, the dermis at the bottom and the stratum corneum at the top, separated by the basal layer. Spectroscopically, these three regions are quite distinguishable, as illustrated by the KMCA mean spectra of figures $4 \mathrm{~B}$, taken within the subsection of figure $4 \mathrm{~A}$ indicated by the black rectangle. Figure $4 \mathrm{C}$ presents the different selected cluster spectra of the skin model which show the clear biochemical differences between the different skin layers. The Raman spectrum in figure 4C (iii) consists almost completely of collagen features and it is therefore identified as the dermis. Assignments of most Raman bands of collagen have been made by Frushour and Koenig ${ }^{45}$. Specific features in the collagen spectrum are two intense bands at $\sim 855 \mathrm{~cm}^{-1}$ and $\sim 938 \mathrm{~cm}^{-1}$. These bands originate from the amino acid side chain vibrations of proline and hydroproline as well as from a $\mathrm{C}-\mathrm{C}$ stretching vibration of the collagen backbone. Proline and hydroxyproline make up about one-fourth of the amino acids in collagen, a higher proportion than in most other proteins ${ }^{46}$. The uppermost layer is identified as the stratum corneum of human skin as the cluster spectrum in figure $4 \mathrm{C}$ (i) is mainly composed of features of keratin and lipids. The position of the amide I band at $1655 \mathrm{~cm}^{-1}$ indicates that keratin in the human stratum corneum adopts predominantly and $\alpha$-helical

conformation ${ }^{47,48}$. The contributions of lipids are normally observed at $1061 \mathrm{~cm}^{-1}, 1128 \mathrm{~cm}^{-1}$ and 
$1296 \mathrm{~cm}^{-1} 46$ but due to tissue processing (formalin fixation, paraffin-embedding) and dewaxing, lipids are largely removed from the samples ${ }^{44}$. The spectrum of the intermediate layer in figure $4 \mathrm{C}$ (ii) contains additional bands which are characteristic of DNA. The bands of the DNA at 1251 $\mathrm{cm}^{-1}$ (guanine, cytosine), $1097 \mathrm{~cm}^{-1}$ (phosphodioxy $\left.\mathrm{PO}_{2}^{-}\right), 811 \mathrm{~cm}^{-1}(\mathrm{O}-\mathrm{P}-\mathrm{O}), \quad 784 \mathrm{~cm}^{-}$ ${ }^{1}$ (phosphodiester, cytosine), $756 \mathrm{~cm}^{-1}$ (DNA), $722 \mathrm{~cm}^{-1}$ (DNA), $666 \mathrm{~cm}^{-1}$ (gunanine, thymine) and $597 \mathrm{~cm}^{-1}$ (nucleotide conformation) are indicative of cellular structures of the skin.

It should be noted that, although the KMCA only identifies two sublayers, the epidermis is divided into four sublayers, the stratum basale or germinativum, stratum spionsum, stratum granulosum and stratum corneum. The major cell type of the epidermis is the keratinocyte, made up of filaments of the protein keratin, providing structural stability to the cell. Keratinocytes differentiate continuously, migrating upward from the basal layer, accumulating keratin. Finally the keratinocoytes are completely cornified and desquamate at the outermost layer. This differentiation takes about 15 to 30 days ${ }^{49-51}$.

Figure 5A shows an optical microscopic image of the dewaxed model skin section, focussing on the epidermal region between the dermis and stratum corneum. A KMCA map of this intermediate region identifies two distinct clusters, largely separated from top to bottom. The cluster spectra, shown as Figure 5C(i) and 5C(ii), respectively, are differentiated by the loss of spectral features associated with nucleic acids in the top layer, indicated by shading. This is consistent with the evolution of the keratinocytes as they progress towards the stratum corneum and associates the lower layer with the stratum basale and the upper layer with the stratum granulosum. 
The stratum basale or germinativum is the deepest layer of the epidermis, separated from the dermis by a continuous basale membrane. The differentiation process of the keratinocoytes starts in the basale layer and cells migrating towards the surface of the skin. In the process of the differentiation, the keratinocytes enter the stratum spinosum that comprise polyhedral cells which have a spine like appearance. The stratum granulosum layer is made up of flattened cells filled with keratohyalin granules and is characterised by anuclear cells. As a natural process of maturation, the cells flatten and lose their nucleus. The keratinocytes in this layer stop dividing and make large amounts of keratin. Finally the keratinocytes have reached the last stage of their maturation and abruptly transform into the flat, fully keratinized, anuclear cells and are described non-viable cornified cells called corneocytes. The differentiated KMCA clusters of Figure 5C are therefore associated with the transition from the DNA rich stratum basale and the DNA deficient stratum spinosum and granulosum layers, while the uppermost layer of Figure 5A (i) is associated with the stratum corneum. Thus, the results presented demonstrate that Raman spectroscopic microscopy can clearly spatially differentiate the different layers of the skin structure, and clearly identify the biochemical characteristics and the evolution of the cellular structure from the basal layer to the stratum corneum.

In the case of FTIR analysis of the skin, while the epidermis and dermis are clearly differentiated, no subclassification of the epidermis is achieved. In general, in comparison to the Raman spectra of human skin, the FTIR spectra are lacking in detail. Further subclustering of the epidermal regions provided no further differentiation of the layer structure. FTIR images were take using a spatial resolution of $6.25 \mu \mathrm{m} \times 6.25 \mu \mathrm{m}$ and a spectral resolution of $4 \mathrm{~cm}^{-1}$, and clearly the superior spatial and spectral resolution of the Raman measurements provide better spatial and spectral definition of the structure of the tissue section. 


\section{ATR Imaging of human skin sections}

A fundamental difference between Raman and IR spectroscopic microscopy is the lateral spatial resolution, determined by the diffraction limit at the wavelengths employed, as well as the imaging optics. For visible sources commonly employed in Raman spectroscopy (wavelength $\sim 0.5 \mu \mathrm{m}$ ), this implies a spot size as low as $1 \mu \mathrm{m}$ diameter is easily attainable. In the mid-IR (wavelength $\sim 5-10 \mu \mathrm{m}$ ), aperture spot sizes of $6.25 \mu \mathrm{m}$ are typical with bench-top instruments. Using Focal Plane Arrays, with individual pixel sizes of smaller than the diffraction limit $(\sim 4$ $\mu \mathrm{m})$, oversampling has been shown to improve the resolution somewhat ${ }^{52}$. As the layer thicknesses in skin are of the order of tens of microns, at such spatial resolution, differentation between the sublayers of the epithelium is quite impossible. KMCA maps of human skin in figures $1 \mathrm{C}$ and $1 \mathrm{D}$ confirm that conventional IR spectroscopic microscopy cannot map the biochemical variations within the epidermal layer of the skin.

The spatial resolution is also governed by the Numerical Aperture of the delivery objective, which can be significantly increased in FTIR microscopy through use of an ATR objective ${ }^{27,28}$. Using a high refractive index element such as germanium, a spatial resolution of down to 1.56 $\mu \mathrm{m} \times 1.56 \mu \mathrm{m}$ can be achieved in ATR mode ${ }^{53}$.

Figure 6A shows an optical microscopic image of a dewaxed hand tissue section obtained using a germanium ATR objective with spatial resolution of $1.56 \mu \mathrm{m}$. Figure $6 \mathrm{~B}$ shows the average IR absorption map using a spectral resolution of $8 \mathrm{~cm}^{-1}$, whereas Figure 6C shows the KMCA map. Each pixel corresponds to $1.56 \mu \mathrm{m}$ a $1.56 \mu \mathrm{m}$ and the overall map area to $195 \mu \mathrm{m} \times 89 \mu \mathrm{m}$. Mapping time was 6 hours, and the RMieS-EMSC preprocessing took $\sim 60$ hours. KMCA cluster analysis of the FTIR-ATR map identifies a $\sim 20 \mu \mathrm{m}$ thick epidermal layer, and the underlying 
dermal layer (Figure 6C). As before, these latter two regions are spectroscopically quite distinguishable, as illustrated by the K-means cluster spectra of figures $6 \mathrm{D}$ and $6 \mathrm{E}$ (fingerprint region only). Similar differences to those observed in Figure 2B are observed in the lipidic peaks in the high frquency range between $2800 \mathrm{~cm}^{-1}$ and $3000 \mathrm{~cm}^{-1}$ and characteristic collagen peaks can be identified in the fingerprint region. Notably, at the higher spatial resolution no superficial "edge" layer is identified, representing an improvement over free space microscopic imaging. However, no further differentiation of the epidermal substructures is obtainable.

ATR-FTIR imaging therefore represents a somewhat improved approach that can allow for the study of morphology and biochemical composition of the skin layers in comparsion to FTIR in transmission mode. However, although the spatial resolution of $1.56 \mu \mathrm{m}$ is close to that of the Raman measurement, the level of spectral detail in Figure 6D remains significantly less than that achieved using Raman, as shown for example in Figure 3C. Notably, in the measurement represented in figure 6 , the spectral resolution was reduced to $8 \mathrm{~cm}^{-1}$, to reduce the sampling time over the larger tissue area. A better comparison between the performance of ATR-FTIR and Raman is shown by the maps in figure 7, for which a spectral resolution of $2 \mathrm{~cm}^{-1}$ was employed.

The two layered structure is again apparent, and the epidermal and dermal clusters are spectroscopically distinct. However, even with a spatial and spectral resolution approaching that of Raman, the ATR-FTIR KMCA cluster spectra are rather similar, and although spectral differnces can be observed, the spectral detail is low compared to Raman and not significantly enhanced compared to the lower resolution measurement of figure 6 .

\section{Discussion}


Although both techniques are widely explored for analysis of tissue and cells and ultimately biomedical applications, comparison of the spectral mapping of human skin tissue and artificial skin model sections demonstrates clear differences between the performances of Raman and infrared spectroscopy. These differences have origin in part in the supporting technologies, but also in the fundamental principles of operation of the two distinct spectroscopic phenomena.

Clearly, in order to spatially resolve the morphological features of the sample of interest, the spatial resolution of the probe should be higher than the size of the features of interest. Although this is limited by the wavelength employed, for a fixed wavelength (range), significant improvements can be achieved through use of high numerical aperture delivery optics, such as those provided by ATR microscopic objectives. For example, ATR-FTIR imaging has been demonstrated capable of mapping the substructures of human hair follicle sections ${ }^{54}$. The improved spatial resolution of the ATR-FTIR mode provides a somewhat improved performance in terms of the edge effects, but does not improve the spatial differentiation of the skin layers, based on their differing biochemical characteristics. In particular, ATR-FTIR does not provide a differentiation of the sublayers of the epithelium, whereas Raman spectroscopy can clearly differentiate between the SC and the underlying epithelium, and in the absence of melanin in the artificial skin model, can even differentiate the sublayers of the basal layer from the overlying epithelium.

In the current study, as tissue sections were examined, no consideration has been given to penetration depth, and indeed the tissue section thickness was chosen to allow measurements in the transmission mode of FTIR. Ultimately, for in vivo applications, penetration depth is an important consideration, however. In Raman spectroscopy, the penetration depth is largely determined by the choice of wavelength of the source laser, and optimally this can be chosen in 
the near infrared region where tissue has a transmission window. Absorption is largely governed by that of melanin (stratum basale) or haemoglobin (sub dermis) across the visible, and by the overtones of $\mathrm{OH}$ vibrations in the near infra red regions. Scattering is an additional loss mechanism, but the development of Spatially Off-Set Raman Spectroscopy ${ }^{55}$, using fibre probe rather than microscope objective delivery and collection optics, has exploited the fact that the signal from the deeper layers is scattered to a greater extent, to improve penetration depth sensitivities. Depth sensitivities of up to several millimeters are now achievable and examples of emerging applications include non-invasive diagnosis of bone disease, cancer and monitoring of glucose levels ${ }^{56}$. As it is intrinsically an absorption technique, however, the penetration depth of FTIR is predominantly limited by the absorption depth in the mid-IR. For a given sample, this depends on the absorption co-efficient at a specific frequency and the density of absorbing medium. The result is that the penetration depth can vary significantly over the spectrum, and, in a physically/chemically inhomogeneous sample, can vary significantly from point to point. This has led to difficulties in interpreting spectra even from single cells, as the nucleus is extremely dense, and almost impenetrable, while the cytoplasm is sparse and relatively transparent ${ }^{57}$. In ATR-FTIR, sampling is made by the evanescent wave of the ATR crystal, and is typically of the order of the wavelength ${ }^{58}$, and although absorbances can be linear in thickness for some hundreds of $\mathrm{nm}^{59}$, the penetration depth in this mode is limited to some microns.

Notably, the complexity of the mean cluster spectra of FTIR images is very much less than that provided by the similar Raman analysis. The spectral resolution of an FTIR measurement is primarily determined by the optical path difference (OPD) traversed by the interferomenter scan, and is usually descibed by $\Delta v=1 / \mathrm{OPD}$. In benchtop instruments, for example the Perkin Elmer Spotlight $400 \mathrm{~N}$, the limit of resolution of $2 \mathrm{~cm}^{-1}$ corresponds to an OPD of $0.5 \mathrm{~cm}$. In Raman 
spectroscopy, the laser source bandwidth is small compared to the resolution determining factors of the dispersion element and the pixel size of the detector. Although a resolution of as low as $0.25 \mathrm{~cm}^{-1}$ is achievable with the LabRam HR800 with high dispersion gratings (1800 lines $\left./ \mathrm{mm}\right)$, the 300 lines/mm grating employed for the measurements shown here produces a dispersion of $\sim 1.5 \mathrm{~cm}^{-1}$ per pixel, comparable to the $2 \mathrm{~cm}^{-1}$ employed for the ATR-FTIR measurements of Figure 7. High Resolution IR spectroscopy is commonly employed for gas phase molecular spectroscopy, and synchrotron sources can allow OPDs of $>10 \mathrm{~m}^{59}$. In this way, bandwidths as low as for example $7 \times 10^{-4} \mathrm{~cm}^{-1}$ for the $v_{18}$ fundamental band of acrolein have been recorded ${ }^{60,61}$. However, while natural spectral bandwidths in the gas phase are broadened principally only by Doppler broadening, in the condensed phase, bandwidths are considerably more perturbed, inhomogeneously, by for example aggregation and other local field effects. Using a resolution of $1.5 \mathrm{~cm}^{-1}$, coupled with a second derivative and curve fitting approach, the Amide I band of lyophilised proteins can be deconvoluted into 6-7 bands which had band widths of $8-28 \mathrm{~cm}^{-1}$ ( http://www.imb-jena.de/ImgLibDoc/ftir/IMAGE_FTIR.html). The substructure of the amide I band yields information on the secondary structure of the proteins. This clearly demonstrates that spectral resolution is not the limiting factor in FTIR analysis of biological samples. Figure $8 \mathrm{~A}$ shows the equivalent amide I region of the average spectra of ATR-FTIR hand skin dermis, taken using $8 \mathrm{~cm}^{-1}$ resolution. Notably, with the exception of the lower noise levels, the trace is indistinguishable from that recorded at $2 \mathrm{~cm}^{-1}$ resolution (Figure $8 \mathrm{~B}$ ). In both cases, although substructure is apparent, resolution enhancement through for example the use of (double) derivatised spectra is required to extract meaningful details. However, in the case of the Raman measurement, taken with the 300 lines $/ \mathrm{mm}$ grating, yielding a dispersion of $\sim 1.5 \mathrm{~cm}^{-1}$ per pixel, the substructure is clearly visible and the as recorded spectra, after baseline correction, can be 
well fitted with 9 Gaussian/Lorentzian bands which provide detail of the protein secondary structures ${ }^{62}$. The fitted band widths range from $7-27 \mathrm{~cm}^{-1}$, in good agreement with the analysis of FTIR bands in (http://www.imb-jena.de/ImgLibDoc/ftir/IMAGE_FTIR.html), but without the requirement of resolution enhancement. Employing the higher resolution of the 1800 lines $/ \mathrm{mm}$ grating, yielding a dispersion of $0.1 \mathrm{~cm}^{-1}$ per pixel, no further detail of the spectra is apparent (data not shown). Clearly, with similar instrumental performance, Raman yields better resolved spectral features which are more easily interpreted.

Although infrared absorption and Raman scattering spectroscopy probe the same physical phenomenon of molecular vibrations, the processes of Raman scattering and infrared absorption are fundamentally different. Indeed the difference is immediately apparent in the different spectral profiles of figure 8 . In both cases, the responses in this amide I region originate primarily in the protein structure of the skin tissue, but the spectral maxima are clearly distinct in the Raman spectrum. Additionally, however, the spectral profile is discernibly richer in substructure in the case of Raman, although the instrumentational specifications are similar in terms of spectral and spatial resolution. Infrared absorption involves an electric dipole transition between two vibrational states, each of which has its own homogeneously and inhomogeneously broadened line width. The resultant spectral bandwidth is a convolution of these two individual line widths. Although often represented as a transition between a real vibrational level of the manifold of an electronic state and a virtual electronic level, Raman is a scattering process. In the representation of the transition to a virtual state, the band width of that state is infinitesimally small, and so the scattering line width is intrinsically less than that of an equivalent infrared absorption transition, giving rise to more distinct spectral features. 
In comparing the application of Raman versus FTIR spectroscopy for biomedical applications, therefore, there are a number of technological and fundamental considerations. It is worth noting a further more practical consideration, that of acquisition and data processing time. Given that the spectral quality is similar, it is valid to compare the $8 \mathrm{~cm}^{-1}$ ATR-FTIR imaging with that of the Raman maps. The ATR - FTIR map of $195 \mu \mathrm{m}$ x $89 \mu \mathrm{m}$ took $\sim 6$ hours to acquire, whereas the comparable Raman map of $36 \mu \mathrm{m} \times 200 \mu \mathrm{m}$ was acquired over $\sim 7 \mathrm{hrs}$. However, the background being significantly reduced in the immersion geometry data preprocessing times were of order $\sim 1$ hour, whereas the 20 iterations RMIE preprocessing took $\sim 60$ hours. This could be reduced by using a lower number of iterations and more powerful computation platforms, but it is notable that the point to point Raman map, with considerably higher spatial and spectral information, including data processing, is complete in a substantially lower timescale. IN the case of the FTIR measurements, it should also be noted however that the acquisition and data processing times could be improved by the use of Focal Plane Array systems and improved correction algorithms (see for example Ref [63]).

\section{Conclusion}

In comparing the spectroscopic modalities of Raman and FTIR spectroscopy for the analysis of the human skin, Raman spectroscopy provides significantly richer spectroscopic detail, upon which a clearer demarcation of the biochemistry of the tissue layers can be made, with similar data acquisition times and significantly lower data processing times. Raman clearly differentiates the three different regions, the epidermis at the top, the dermis at the bottom, separated by the combined basal and malpighian layers of the epidermis. In the case of the artificial skin model, it can further differentiate the sub layers of the epithelium. However, FTIR differentiates only the 
epidermis and the dermis. Improved spectral and spatial resolution do not significantly improve the performance of FTIR, and it is concluded that the intrinsically higher spectral resolution of Raman, as a scattering rather than resonant absorption process, are fundamental to the differing performances.

\section{Acknowledgements}

This research was supported by the National Biophotonics and Imaging Platform (NBIP) Ireland, Higher Education Authority PRTLI (Programme for Research in Third Level Institutions) Cycle 4, co-funded by the Irish Government and the European Union Structural Fund. 


\section{Figure Legends:}

Figure 1: (A) Optical image of human hand skin tissue section; (B) Infrared image of human hand skin tissue section; (C) KMCA map of human hand skin tissue section; (D) KMCA map of human hand skin tissue section scattering using RMieS-EMSC after 20 iterations

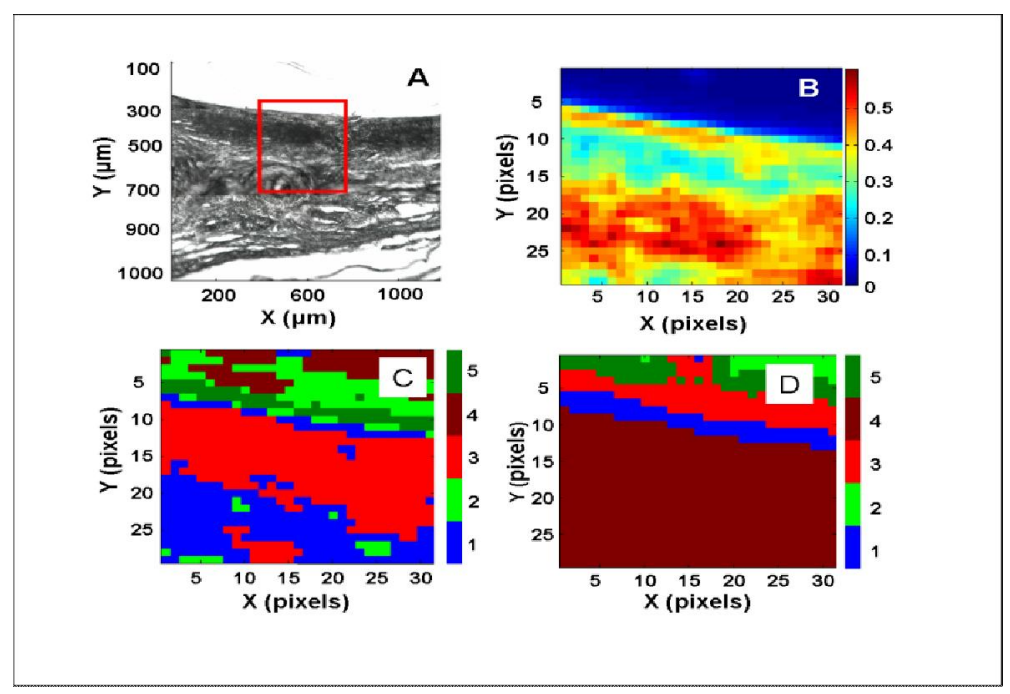

Figure 2: (A) KMCA mean spectra of (i) cluster 5, (ii) cluster 3 and (iii) cluster 1; (B) KMCA mean spectra of (i) cluster 3, (ii) cluster 1 and cluster 4 (iii) cluster using RMieS-EMSC after 20 iteration; (C) KMCA mean spectra of human skin hand in the fingerprint region $\left(1000 \mathrm{~cm}^{-1}\right.$ to $1800 \mathrm{~cm}^{-1}$ ) (i) cluster 4, (ii) cluster 1 and (iii) cluster 3 


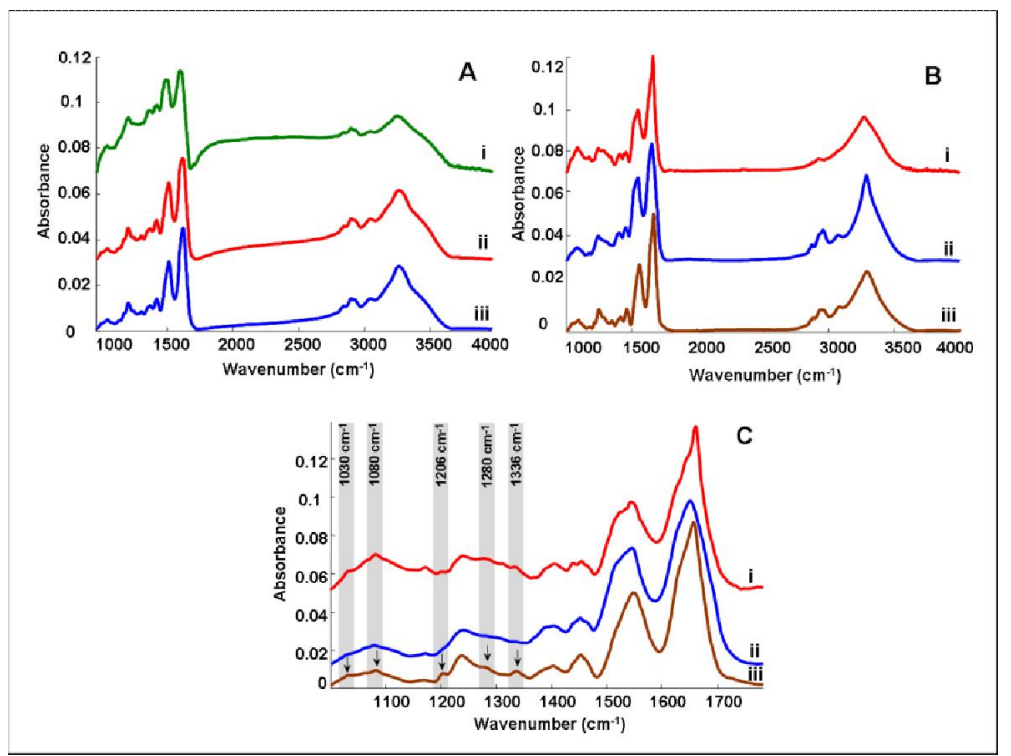

Figure 3: (A) Optical image of human hand skin tissue section; (B) KMCA of Raman spectral map; (C) KMCA mean Raman spectra (i) cluster 3, stratum corneum (ii) cluster 5, intermediate epithelial layer (iii) cluster 4, dermis

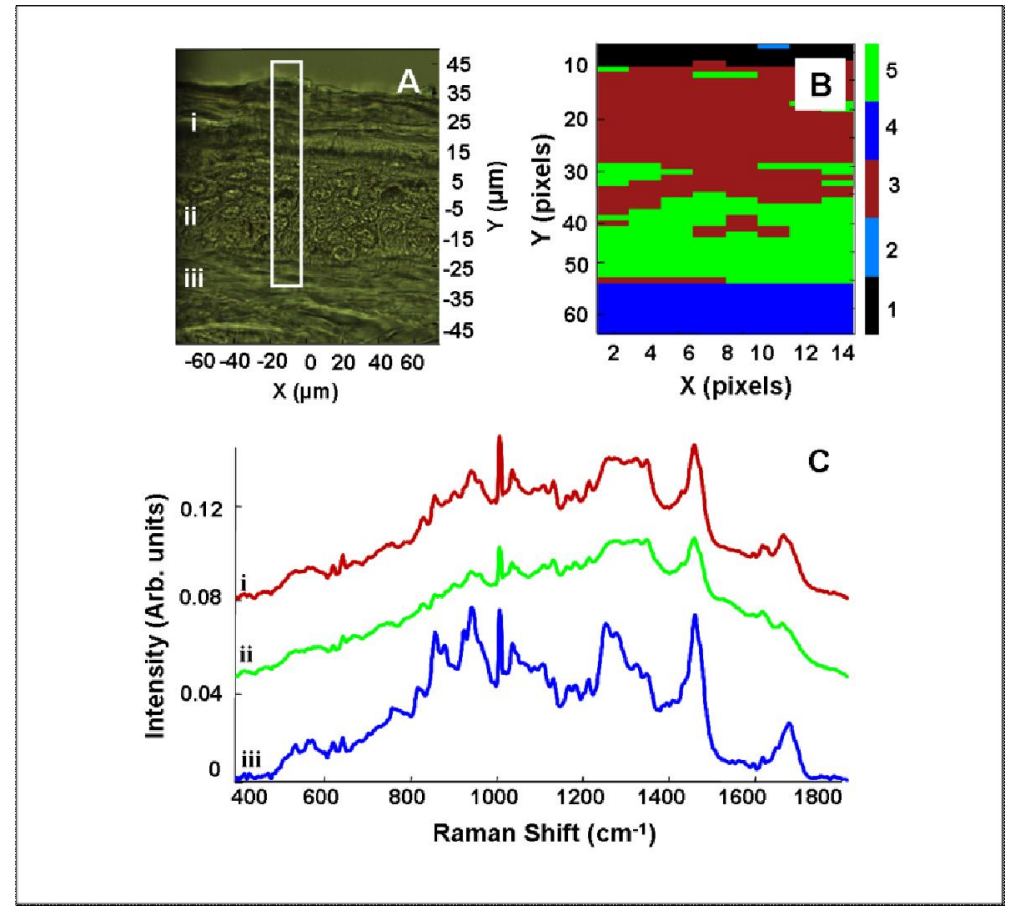


Figure 4: (A) Optical image of artifical tissue section; (B) KMCA of Raman spectral map; (C) KMCA mean Raman spectra of artifical skin tissue (i) cluster 3, stratum corneum (ii) cluster 4, basal layer (iii) cluster 2, dermis

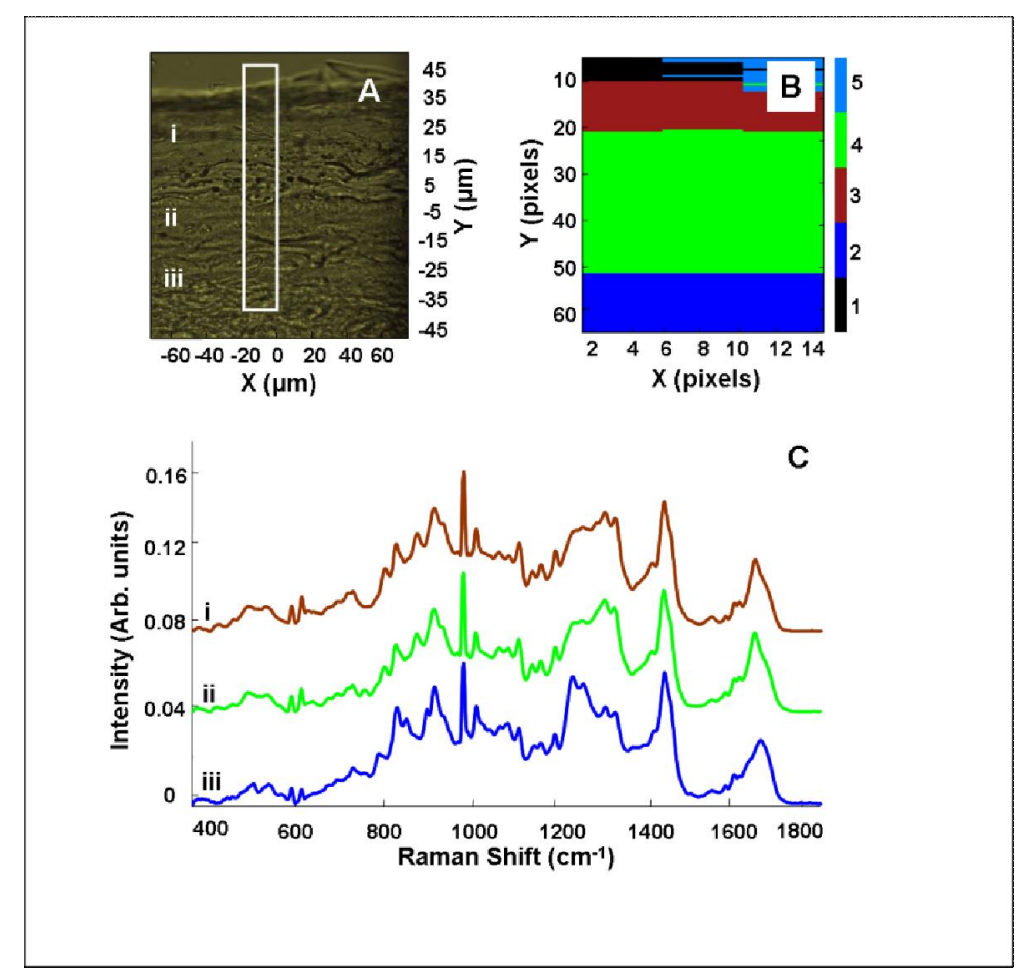

Figure 5: (A) Optical image of artifical tissue section (intermediate epithelial layer); (B) KMCA of Raman spectral map of artfical skin tissue (intermediate epithelial layer); (C) KMCA mean Raman spectra of artifical skin tissue section (intermediate layer) (i) cluster 1 stratum basale (ii) cluster 2 stratum granulosum 


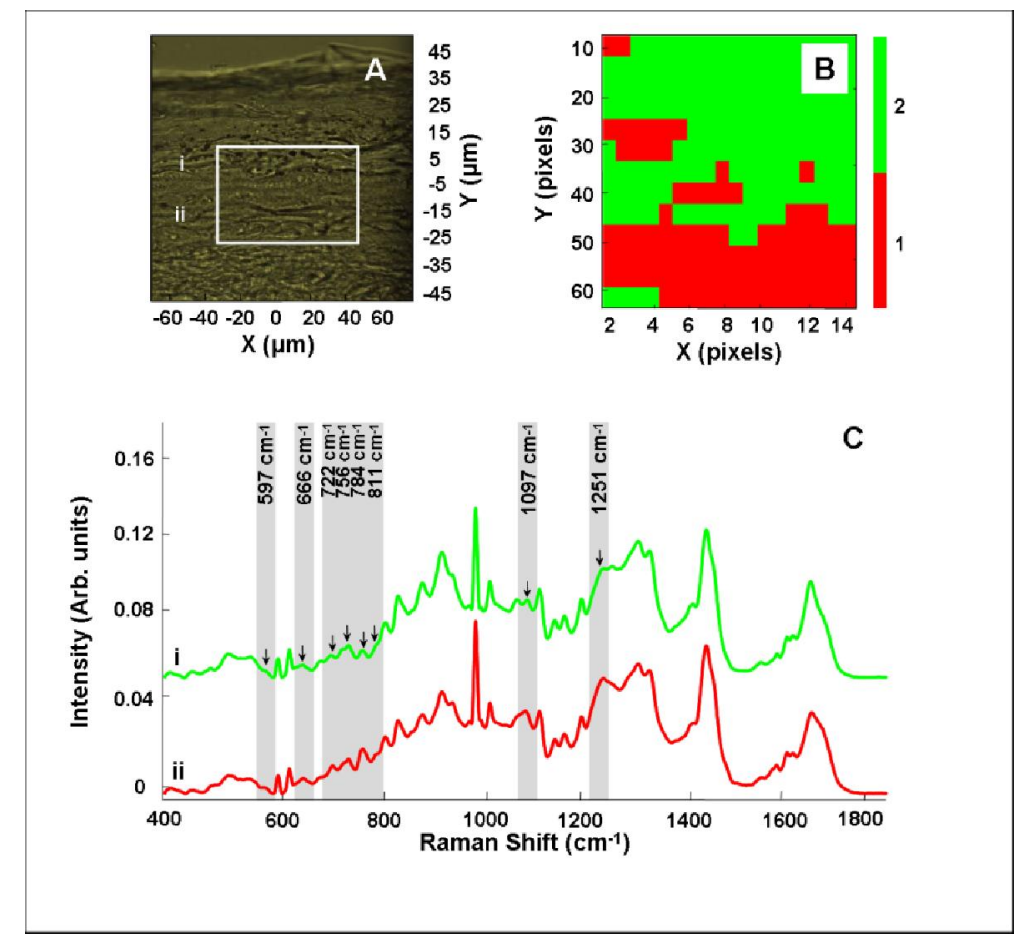

Figure 6: (A) ATR Optical image of human hand skin; (B) ATR image of human hand skin; (C) KMCA means of the ATR map; (D) ATR spectra of human hand skin from top to bottom: (i) cluster 1 hand epidermis, (ii) cluster 4 hand dermis; (E) Fingerprint region of (i) cluster 1 hand epidermis, (ii) cluster 4 hand dermis 


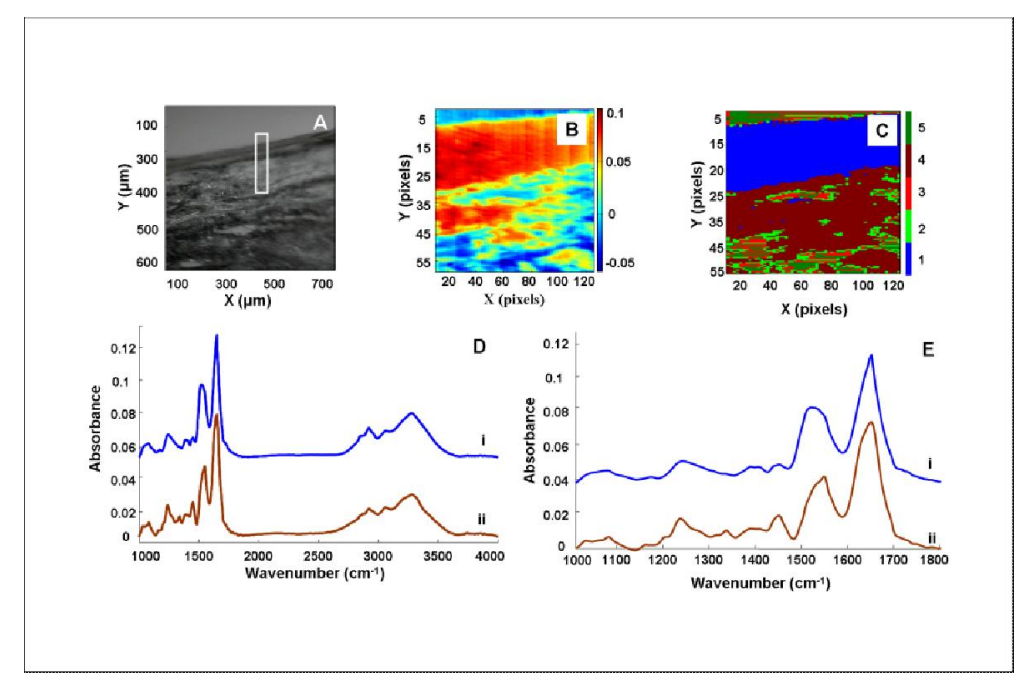

Figure 7: (A) ATR Optical image of human hand skin; (B) ATR average FTIR image of human hand skin; (C) KMCA means ATR map; (D) ATR spectra of human hand skin from top to bottom: (i) cluster 2 hand epidermis, (ii) cluster 5 dermis; (E) Fingerprint region of (i) cluster 2 hand epidermis, (ii) cluster 4 hand dermis

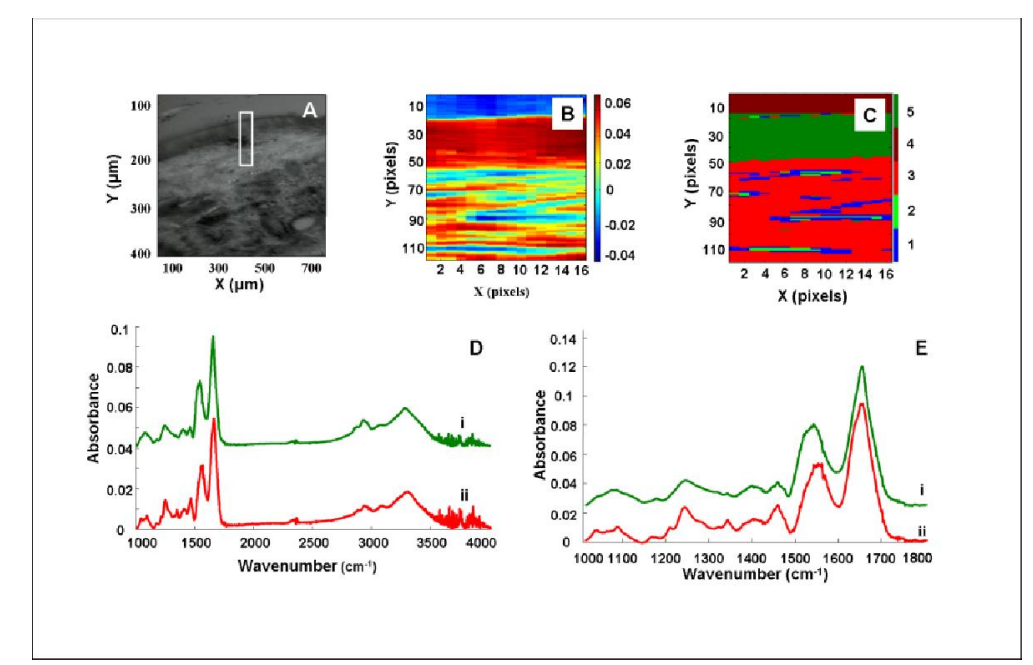


Figure 8: Amide I region of dermal layer of human skin recorded with (A) ATR-FTIR with 8 $\mathrm{cm}^{-}$ 1 spectral resolution; (B) ATR-FTIR with $2 \mathrm{~cm}^{-1}$ spectral resolution; (C) Raman with 300 lines $/ \mathrm{mm}$ grating $\left(1.5 \mathrm{~cm}^{-1}\right.$ per pixel $)$

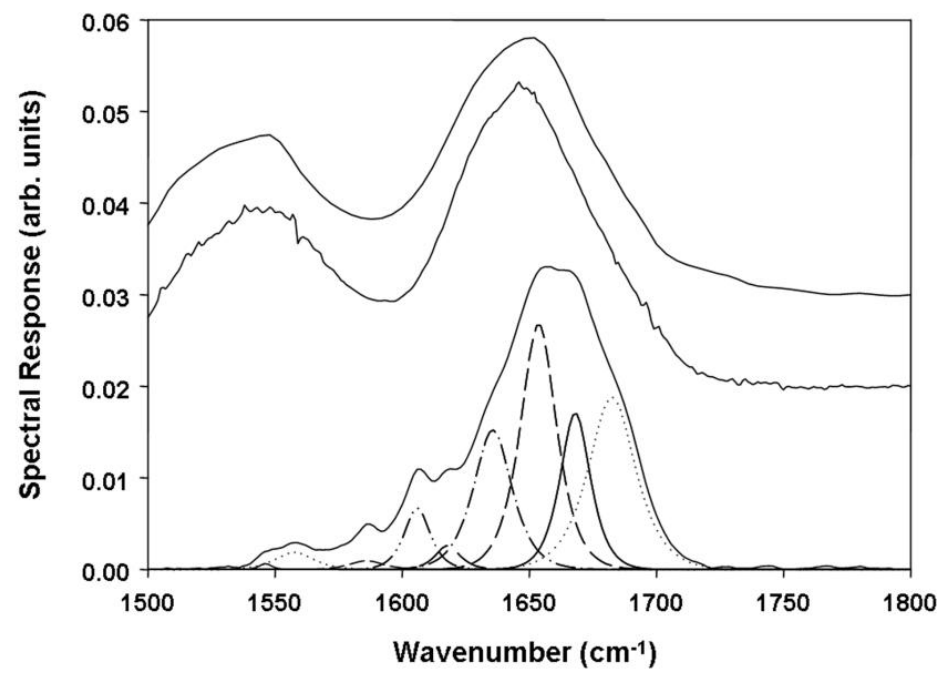




\section{References}

1. Draux F, Jeannesson P, Beljebbar A, Tfayli A, Fourre N, Manfait M, Sule-Suso J, Sockalingum GD, Analyst, 2009, 134, 542-548.

2. Mahadevan-Jansen A, Mitchell MF, Ramanujam N, Malpica A, Thomsen S, Utzinger U, Richards-Kortum R, Photochem Photobiol, 1998, 68, 123-132.

3. Utzinger U, Brewer M, Silva E, Gershenson D, Blast RC, Jr., Follen M, Richards-Kortum R, Lasers Surg Med, 2001, 28, 56-66.

4. Gniadecka M, Faurskov Nielsen O, Christensen DH, Wulf HC, J Invest Dermatol, 1998, 110, 393-398.

5. Nunes LD, Martin AA, Silveira L, Zampieri M, Spectroscopy-an International Journal, 2003, 17, 597-602.

6. Nijssen A, Bakker Schut TC, Heule F, Caspers PJ, Hayes DP, Neumann MH, Puppels GJ, J Invest Dermatol, 2002, 119, 64-69.

7. Caspers PJ, Lucassen GW, Carter EA, Bruining HA, Puppels GJ, J Invest Dermatol, 2001, 116, 434-442.

8. Kaminaka S, Yamazaki H, Ito T, Kohda E, Hamaguchi H, Journal of Raman Spectroscopy, 2001, 32, 139-141.

9. Huang Z, McWilliams A, Lui H, McLean DI, Lam S, Zeng H, Int J Cancer, 2003, 107, 1047 1052.

10. Mizuno A, Kitajima H, Kawauchi K, Muraishi S, Ozaki Y, Journal of Raman Spectroscopy 1994, 25, 25-29.

11. Boere IA, Robinson DJ, de Bruijn HS, van den Boogert J, Tilanus HW, Sterenborg HJ, de Bruin RW, Photochem Photobiol, 2003, 78, 271-277.

12. Kendall C, Stone N, Shepherd N, Geboes K, Warren B, Bennett R, Barr H, J Pathol, 2003, 200, 602-609.

13. Molckovsky A, Song LM, Shim MG, Marcon NE, Wilson BC, Gastrointest Endosc, 2003, 57, 396-402.

14. Crow P, Stone N, Kendall CA, Uff JS, Farmer JA, Barr H, Wright MP, Br J Cancer, 2003, 89, 106-108.

15. Lau DP, Huang Z, Lui H, Man CS, Berean K, Morrison MD, Zeng H, Lasers Surg Med, 2003, 32, 210-214.

16. Lau DP, Huang Z, Lui H, Anderson DW, Berean K, Morrison MD, Shen L, Zeng H, Lasers Surg Med, 2005, 37, 192-200.

17. Krishna CM, Sockalingum GD, Kurien J, Rao L, Venteo L, Pluot M, Manfait M, Kartha VB, Appl Spectrosc, 2004, 58, 1128-1135.

18. Frank CJ, McCreery RL, Redd DC, Anal Chem, 1995, 67, 777-783.

19. Shafer-Peltier KE, Haka AS, Fitzmaurice M, Crowe J, Myles J, Dasari RR, Feld MS, Journal of Raman Spectroscopy, 2002, 33, 552-563.

20. Hawi SR, Campbell WB, Kajdacsy-Balla A, Murphy R, Adar F, Nithipatikom K, Cancer Lett, 1996, 110, 35-40.

21. Notingher I, Verrier S, Haque S, Polak JM, Hench LL, Biopolymers, 2003, 72, 230-240.

22. Swain RJ, Jell G, Stevens MA, Journal of Cellular Biochemistry, 2008, 104, 1427-1438.

23. Bonnier F, Knief P, Lim B, Meade AD, Dorney J, Bhattacharya K, Lyng FM, Byrne HJ, Analyst, 2010, 135, 3169-3177.

24. Dorney J, Bonnier F, Garcia A, Casey A, Chambers G, Byrne HJ, Analyst, 2012, 137, 1111-1119. 
25. Tfayli A, Piot O, Durlach A, Bernard P, Manfait M, Biochim Biophys Acta, 2005, 1724, 262 -

269.

26. Bonnier N, Schmitt F, Leynadier C, in Proceedings of the14th IS\&T/SID Color Imaging

Conference, vol. 1, (Scottsdale, Arizona)

2009, 341-346.

27. Sommer AJ, Tisinger LG, Marcott C, Story GM, Applied Spectroscopy, 2001, 55, 252-256.

28. Ippolito S. B., Goldberg B. B., Ünlü M. S., Appl. Phys. Lett. 78, 40712001.

29. Byrne HJ, Sockalingum GD, Stone N, in "Biomedical Applications of Synchrotron Infrared Microspectroscopy: A Practical Approach", David Moss, (Editor), RSC Analytical Spectroscopy Monographs No. 11 (2011) ISBN: 978-0-85404-154-1

30. Romeo M, Diem M, Vibrational Spectroscopy, 2005, 38, 129-132.

31. Romeo M, Mohlenhoff B, Diem M, Vibrational Spectroscopy, 2006, 42, 9-14.

32. Romeo MJ, Diem M, Vibrational Spectroscopy, 2005, 38, 115-119.

33. Lee J, Gazi E, Dwyer J, Brown MD, Clarke NW, Nicholson JM, Gardner P, Analyst, 2007, 132, 750-755.

34. Bassan P, Byrne HJ, Bonnier F, Lee J, Dumas P, Gardner P, Analyst, 2009, 134, 1586-1593.

35. Bassan P, Byrne HJ, Lee J, Bonnier F, Clarke C, Dumas P, Gazi E, Brown MD, Clarke NW, Gardner P, Analyst, 2009, 134, 1171-1175.

36. Bassan P, Kohler A, Martens H, Lee J, Byrne HJ, Dumas P, Gazi E, Brown M, Clarke N, Gardner P, Analyst, 2010, 135, 268-277.

37. Bassan P, Kohler A, Martens H, Lee J, Jackson E, Lockyer N, Dumas P, Brown M, Clarke N, Gardner P, Journal of Biophotonics, 2010, 3, 609-620.

38. Kohler A, Sule-Suso J, Sockalingum GD, Tobin M, Bahrami F, Yang Y, Pijanka J, Dumas P, Cotte M, van Pittius DG, Parkes G, Martens H, Applied Spectroscopy, 2008, 62, 259-266.

39. Fowler LJ, Lachar WA, Arch Pathol Lab Med, 2008, 132, 373-383.

40. Hughes C, Liew M, Sachdeva A, Bassan P, Dumas P, Hart CA, Brown MD, Clarke NW, Gardner P, Analyst, 2010, 135, 3133-3141.

41. Krafft C, Codrich D, Pelizzo G, Sergo V, Journal of Biophotonics, 2008, 1, 154-169.

42. Koljenovic S, Schut TCB, van Meerbeeck JP, Maat A, Burgers SA, Zondervan PE, Kros JM, Puppels GJ, Journal of Biomedical Optics, 2004, 9, 1187-1197.

43. Ly E, Piot O, Durlach A, Bernard P, Manfait M, Analyst, 2009, 134, 1208-1214.

44. Ali SM, Bonnier F, Tfayli A, Lambkin H, Flynn K, McDonagh V, Healy C, Lee TC, Lyng FM, Byrne HJ, Journal of Biomedical Optics, 2013.

45. Frushour BG, Koenig JL, Biopolymers, 1975, 14, 379-391.

46. Caspers PJ, Lucassen GW, Wolthuis R, Bruining HA, Puppels GJ, Biospectroscopy, 1998, 4, S31-39.

47. Anigbogu ANC, Williams AC, Barry BW, Edwards HGM, International Journal of Pharmaceutics, 1995, 125, 265-282.

48. Potts RO, Guzek DB, Harris RR, McKie JE, Arch Dermatol Res, 1985, 277, 489-495.

49. Mendelsohn BA, Yin C, Johnson SL, Wilm TP, Solnica-Krezel L, Gitlin JD, Cell Metabolism, 2006, 4, 155-162.

50. Poumay Y, Coquette A, Archives of Dermatological Research, 2007, 298, 361-369.

51. Martini FH, Bartholomew EF, Essentials of Anatomy and Physiology, 2007, Benjamin Cummins Publishing, San Francisco, ISBN 9781405854863

52. Reddy RK, Walsh MJ, Schulmerich MV, Carney PS, Bhargava R, (2013) Applied Spectroscopy, 67, 93-105

53. Walsh MJ, Holton SE, Kajdacsy-Balla A, Bhargava R, Vibrational Spectroscopy, 2012, 60, 23 -

28.

54. Chan KLA, Kazarian SG, Mavraki A, Williams DR, Applied Spectroscopy, 2005, 59, 149-155. 
55. Matousek P, Clark IP, Draper ERC, Morris MD, Goodship AE, Everall N, Towrie M, Finney WF and Parker AW, 2005, Applied spectroscopy 59, 393-400

56. Matousek P and Stone N, 2013, J. Biophotonics 6, 7-19

57. Mohlenhoff B, Romeo M, Diem M and Wood BR, 2005 Biophysical Journal 88(5) 3635-3640

58. Harrick NR, 1967, Internal Reflection Spectroscopy, Wiley/Interscience, New York.

59. Kane SR, Ashby PD, Pruitt LA, 2009, Journal of Biomedical Materials Research Part B: Applied Biomaterials, 91, 613-620

59. McKellar ARW, Journal of Molecular Spectroscopy, 2010, 262, 1-10.

60. McKellar ARW, Appadoo DRT, Journal of Molecular Spectroscopy, 2008, 250, 106-113.

61. McKellar ARW, Tokaryk DW, Li-Hong Xu, Appadoo DRT, May T, Journal of Molecular Spectroscopy, 2007, 242, 31-38.

62. Kinalwa MN, Blanch EW, Doig AJ, Analytical Chemistry, 2010, 82, 6347-6349.

63. Bird B, Miljkovic M, Diem M, 2010, Journal of Biophotonics, 3, 597-608 